\title{
Teste de Condutividade Elétrica em Sementes de Bowdichia virgilioides Kunth
}

\author{
Samanta Jaqueline Dalanhol ${ }^{1}$, Eduardo Henrique Rezende ${ }^{2}$, \\ Daniela Cleide Azevedo de Abreu ${ }^{3}$, Antonio Carlos Nogueira ${ }^{2}$
}

\begin{abstract}
${ }^{1}$ Departamento de Botânica, Universidade Estadual Paulista "Júlio de Mesquita Filho" - UNESP, Botucatu/SP, Brasil
${ }^{2}$ Departamento de Ciências Florestais, Universidade Federal do Paraná - UFPR, Curitiba/PR, Brasil

${ }^{3}$ Coordenação de Engenharia Florestal, Universidade Tecnológica Federal do Paraná - UTFPR, Dois Vizinhos/PR, Brasil
\end{abstract}

\section{RESUMO}

O objetivo deste trabalho foi adequar a metodologia do teste de condutividade elétrica (CE), além de determinar a qualidade fisiológica de sementes de B. virgilioides. As sementes foram separadas em três sublotes, de acordo com a coloração do tegumento (1-amarela, 2-alaranjada e 3 -vermelha/preta). No teste de CE, estudaram-se três quantidades de sementes $(25,50100)$, três volumes de água $(25,50$ e $100 \mathrm{~mL})$ e seis tempos de embebição das sementes $(12,24,36$, 48, 60 e $72 \mathrm{~h}$ ), a $25^{\circ} \mathrm{C}$, com seis repetições. Para comparação dos resultados, também se realizou o teste de germinação. O teste de CE possibilitou a diferenciação dos sublotes em três níveis de vigor, igualmente ao observado pelo teste de germinação, sendo que as sementes amarelas possuem o maior vigor, seguidas pelas sementes alaranjadas e vermelhas/pretas, com menor vigor. Recomenda-se utilizar 25, 50 ou 100 sementes embebidas em $50 \mathrm{~mL}$ de água por 24 horas.

Palavras-chave: sucupira-preta, testes de vigor, maturação fisiológica.

\section{Electrical Conductivity Test in Seeds of Bowdichia virgilioides Kunth}

\begin{abstract}
The aim of this study was to adapt the methodology of electrical conductivity (EC) test and determine the physiological quality of seeds of $B$. virgilioides. Seeds were separated into three batches according to tegument color (1-yellow, 2-orange, and 3-red/black). The EC test analyzed three quantities of seeds $(25,50$ and 100$)$, three volumes of water $(25,50$ and $100 \mathrm{~mL})$, and six times of seed imbibition $(12,24,36,48,60$ and 72$)$, at $25{ }^{\circ} \mathrm{C}$ with 6 replications. Germination test was employed to compare the results. The EC test allowed the differentiation of batches in three levels of vigor - the same was observed in germination test: batch 1 presented the best seed vigor, followed by batches 2 and 3. The use of 25, 50 or 100 seeds imbibed in $50 \mathrm{~mL}$ of water for 24 hours is recommended.
\end{abstract}

Keywords: black sucupira, seed vigor tests, physiological maturation. 


\section{INTRODUÇÃO}

Bowdichia virgilioides Kunth, da família Fabaceae, é conhecida popularmente como sucupira-parda, sucupira-roxa, sucupira-preta, sucupira-do-cerrado ou paricarana, e caracterizase por ser uma espécie pioneira, decídua e heliófita. Não tolera baixas temperaturas e pode alcançar, na idade adulta, até $15 \mathrm{~m}$ de altura, com $60 \mathrm{~cm}$ de DAP. Ocorre principalmente no Cerrado, adaptando-se bem em solos secos e de baixa fertilidade, sendo recomendada para uso medicinal, no paisagismo e em recomposição de áreas degradadas. Também possui madeira de ótima qualidade, com resistência ao ataque de organismos xilófagos (Carvalho, 2006; Lorenzi, 2008; Matos \& Queiroz, 2009).

As sementes desta espécie possuem dormência tegumentar por impermeabilidade à entrada de água, dificultando sua germinação; esta pode variar de $45 \%$ a $88 \%$, com superação da dormência, e de $0 \%$ a $2,1 \%$, sem tratamentos pré-germinativos (Carvalho, 2006). $\mathrm{O}$ fato de as sementes apresentarem dormência pode ser um fator de atraso na determinação da sua qualidade fisiológica, demonstrando a importância de se utilizarem testes que determinem o vigor das sementes em um intervalo menor de tempo.

Para a produção de mudas por sementes, a padronização e o aperfeiçoamento do teste de germinação visam a avaliar o potencial das sementes para semeadura e comparar a qualidade de diferentes lotes, o que deve ser conduzido sob condições ambientais ideais e específicas para cada espécie (Brasil, 2009). Apesar de ser rotineiramente utilizado em laboratórios de análises de sementes, o teste de germinação requer períodos de tempo relativamente longos para sua execução. Os testes de avaliação rápida das sementes, como o teste de condutividade elétrica, permitem agilizar a obtenção de informações (Tillmann \& Miranda, 2007), como também fornecem respostas complementares às fornecidas pelo teste de germinação em prazo relativamente curto (Gonçalves et al., 2008).

O teste de condutividade elétrica está diretamente relacionado à integridade das membranas celulares. A habilidade de reorganização e reparação dos danos pelas membranas celulares é maior em sementes com maior vigor, em comparação com sementes de baixo vigor. Quanto maior o vigor das sementes, mais rapidamente estas irão reparar os danos da membrana, diminuindo os eletrólitos lixiviados para o meio externo (Vieira \& Krzyzanowski, 1999).

Neste teste, o vigor é avaliado indiretamente, ou seja, as diferenças entre os valores das leituras de condutividade de diferentes amostras indicam possíveis diferenças no vigor das sementes. Assim, as sementes com baixo vigor, com sistema de membranas desorganizado, liberarão mais eletrólitos, aumentando a condutividade elétrica da solução de embebição (Marcos Filho, 2005).

Após o beneficiamento das sementes, estas podem ser classificadas de acordo com peso específico, forma, textura do tegumento, cor ou afinidade a líquidos (Fowler \& Martins, 2001). As sementes de $B$. virgilioides apresentam coloração variando de tons castanhos escuros a avermelhados e ferrugíneos (Carvalho, 2006). Estas diferentes colorações do tegumento podem ser encontradas dentro do mesmo fruto e acredita-se que isto esteja associado ao grau de maturação das sementes, podendo influenciar no seu potencial fisiológico.

Considerando-se a escassez de pesquisas que relacionam a cor do tegumento aos graus de maturação das sementes, o objetivo deste trabalho foi adequar a metodologia do teste de condutividade elétrica para sementes de sucupira-preta, variandose a quantidade de sementes, os volumes de água e os períodos de embebição, como também determinar a qualidade fisiológica de três lotes de sucupira-preta separados de acordo com a coloração do tegumento.

\section{MATERIAL E MÉTODOS}

Os frutos de B. virgilioides foram coletados de dez matrizes (com, no mínimo, 200 metros de distância entre cada uma), localizadas no município de Ipameri, Goiás, pertencente ao domínio fitogeográfico do Cerrado. Em seguida, as sementes foram transferidas para o Laboratório de Sementes Florestais da Universidade Federal do Paraná, onde o experimento foi conduzido.

Anteriormente à instalação do teste de condutividade elétrica, efetuaram-se as análises físicas das sementes. Determinou-se o peso de mil sementes, no qual foram utilizadas oito repetições de 
100 sementes, seguindo as instruções determinadas pelas Regras para Análises de Sementes (Brasil, 2009). Realizou-se, também, a determinação do grau de umidade com três repetições de aproximadamente cinco gramas de sementes intactas, as quais permaneceram em estufa a $105{ }^{\circ} \mathrm{C} \pm 3{ }^{\circ} \mathrm{C}$ por 24 horas. Os resultados foram expressos com base no peso úmido das sementes, de acordo com Brasil (2009).

Realizou-se a análise de pureza, separando-se o lote em sementes puras (intactas) e material inerte, incluindo as sementes danificadas por insetos e o material orgânico resultante do beneficiamento. A pureza do lote foi determinada segundo Brasil (2009). A porção de sementes puras foi dividida em três sublotes de acordo com a coloração do tegumento: amarela, alaranjada e vermelha/preta. As sementes vermelhas e pretas foram mantidas juntas, pois se apresentavam em menor quantidade no lote inicial.

O teste de germinação foi realizado segundo um delineamento inteiramente casualizado, com seis repetições de 25 sementes por tratamento (sublote). Para a superação da dormência tegumentar, fez-se um corte superficial com tesoura na região oposta à micrópila, para facilitar a entrada deágua. As sementes foram distribuídas em caixas gerbox com papel mata-borrão umedecido com 2,5 vezes o seu peso; em seguida, foram transferidas para germinadores, nos quais permaneceram à temperatura de $25^{\circ} \mathrm{C}$. Foram consideradas germinadas as sementes que apresentaram raiz primária maior ou igual a $2 \mathrm{~mm}$ (Hadas, 1976). As contagens foram realizadas diariamente até a estabilização da germinação, que ocorreu aos dez dias.

As variáveis avaliadas foram porcentagem de germinação, tempo médio de germinação (TMG) (Labouriau, 1983) e índice de velocidade de germinação (Maguire, 1962). Os dados foram submetidos ao teste de Bartlett para testar as variâncias dos tratamentos quanto à homogeneidade: as que se mostraram homogêneas foram submetidas ao teste $\mathrm{F}$ e, pelo teste de Tukey, suas médias foram comparadas em nível de $5 \%$ de probabilidade. Os dados não homogêneos (porcentagem de germinação) foram trasformados em arco seno $\sqrt{\frac{x}{100}}$. Os dados transformados foram utilizados somente na análise estatística, enquanto que, nas tabelas, mantiveram-se os dados originais.

Para o teste de condutividade elétrica, utilizaramse três sublotes, sendo: sublote 1 , as sementes amarelas; sublote 2 , as sementes alaranjadas, e sublote 3 , as sementes vermelhas ou pretas. A partir destes sublotes, estudaram-se três volumes de água $(25,50$ e $100 \mathrm{~mL})$, três quantidades de sementes $(25$, 50 e 100 sementes) e seis tempos de embebição em água (12, 24, 36, 48, 60 e 72 horas). Utilizaram-se seis repetições para cada combinação de tratamento, sendo as sementes mantidas em recipientes plásticos com tampa (capacidade: $100 \mathrm{~mL}$; diâmetro de base: 5,6-6 cm), em BOD à temperatura de $25^{\circ} \mathrm{C}$.

Após cada período, fez-se a leitura da solução de embebição, utilizando um condutivímetro Digimed DM-3, sendo o resultado da leitura dividido pelo peso da subamostra, expresso em $\mu \mathrm{S} \mathrm{cm}^{-1} \mathrm{~g}^{-1} \mathrm{de}$ semente.

O delineamento experimental foi inteiramente casualizado, em parcelas subdivididas no tempo, sendo os dados da condutividade elétrica (CE) analisados separadamente, para cada combinação entre quantidades de sementes e volumes de água.

Utilizou-se o teste de Bartlett para testar as variâncias dos tratamentos quanto à homogeneidade: as que se mostraram homogêneas, foram submetidas ao teste $\mathrm{F}$ e, pelo teste de Tukey, suas médias foram comparadas a $5 \%$ de probabilidade. Os dados não homogêneos foram transformados em Log (X). Utilizou-se o programa estatístico Assistat 7.6 Beta (2012). Para observar a tendência dos valores de condutividade elétrica com o aumento do tempo de embebição, procedeu-se ao estudo de regressão polinomial, com o programa Microsoft Office Excel 2007.

\section{RESULTADOS E DISCUSSÃO}

O lote inicial de sementes de B. virgilioides apresentou $8,95 \%$ de umidade. De acordo com Vieira \& Krzyzanowski (1999), os lotes de sementes submetidos ao teste de condutividade elétrica devem apresentar valores uniformes de umidade, pois algum lote com umidade muito baixa em relação aos outros pode ser subestimado no teste. Tal característica não foi observada no presente estudo, pois os três 
sublotes de sucupira-preta são provenientes de um lote inicial com único teor de água.

De acordo com a porcentagem de germinação, observam-se, na Tabela 1, diferenças significativas de viabilidade entre as três amostras de sementes de sucupira-preta analisadas. As sementes amarelas apresentaram maior porcentagem de germinação (98\%), seguidas pelas sementes alaranjadas e pelas sementes vermelhas/pretas, as quais apresentaram os valores de $85,3 \%$ e $31,3 \%$, respectivamente. Isto mostra que a porcentagem de germinação foi eficiente na diferenciação das amostras de sementes. De acordo com Borghetti \& Ferreira (2004), sementes com porcentagem de germinação semelhante podem ter comportamentos germinativos diferentes, ao contrário do encontrado no presente trabalho, pois o TMG e o IVG não mostraram diferenças significativas entre as três amostras, ou seja, não foram bons para detectar diferenças de vigor entre estas.

Para Gonçalves et al. (2008), a redução do índice de velocidade de germinação não está relacionada com o início do processo de deterioração das sementes, porém esse índice tem sido bastante utilizado, apresentando resultados coerentes com o potencial fisiológico das sementes. Isto não foi observado neste estudo, visto que, para o IVG, as sementes vermelhas/pretas apresentaram o menor valor, seguidas pelas amarelas e alaranjadas, que foram estatisticamente iguais (Tabela 1).

Tabela 1. Valores de porcentagem de germinação (\%G), índice de velocidade de germinação (IVG), tempo médio de germinação (TMG), coeficiente de variação (CV\%) e quadrados médios para diferentes sublotes de sementes de Bowdichia virgilioides Kunth.

Table 1. Values of germination percentage (\%G), germination speed index (IVG), mean germination time (TMG), coefficient of variation (CV\%) and F test for different seed lots Boudichia virgilioides Kunth.

\begin{tabular}{|cccc|}
\hline & \%G & IVG & TMG \\
\hline Amarela & $98,00 \mathrm{a}$ & $4,81 \mathrm{a}$ & $5,36 \mathrm{a}$ \\
\hline Alaranjada & $85,30 \mathrm{~b}$ & $4,33 \mathrm{a}$ & $5,15 \mathrm{a}$ \\
\hline Vermelha/Preta & $31,30 \mathrm{c}$ & $1,76 \mathrm{~b}$ & $4,51 \mathrm{a}$ \\
\hline CV $(\%)$ & 4,17 & 20,26 & 12,72 \\
\hline Quadrados médios & $4,63^{* *}$ & $16,15^{* *}$ & $0,33^{\text {ns }}$ \\
\hline
\end{tabular}

Médias seguidas pela mesma letra na coluna não diferem estatisticamente pelo teste de Tukey a $5 \%$ de probabilidade. ** Significativo a $1 \%$. ${ }^{\text {ns }}$ Não significativo.
O teste de condutividade elétrica foi eficiente na diferenciação dos sublotes, apresentando resultados semelhantes aos do teste de germinação, sendo as sementes amarelas de melhor vigor, seguidas pelas alaranjadas e vermelhas/pretas. Estas últimas apresentaram valores significativamente elevados de $\mathrm{CE}$ em relação aos demais. Esta diferenciação nos sublotes foi verificada em todos os tratamentos, desde as 12 horas de embebição (Tabela 2).

Este teste também tem se mostrado eficiente na determinação da qualidade fisiológica de outras espécies florestais, tais como Dalbergia nigra (Marques et al., 2002), Sebastiania commersoniana (Santos \& Paula, 2005), Senna siamea (Dutra et al., 2007), Dictyoloma vandellianum (Flávio \& Paula, 2010), Jatropha curcas (Araujo et al., 2011), Pterogyne nitens (Ataide et al., 2012) e Solanum sessiliflorum (Pereira \& Martins Filho, 2012).

Este padrão no aumento da CE decorrente da diminuição do vigor não é observado em todos os trabalhos realizados. Para a espécie Albizia hassleri, o teste não foi eficiente na discriminação dos lotes analisados (Gonzales et al., 2009). Para Guazuma ulmifolia, o teste mostrou-se menos adequado para a determinação do potencial fisiológico das sementes (Gonçalves et al., 2008) e, nas sementes de Psidium cattleianum (Silva et al., 2011) e nos diásporos de Myracrodruon urundeuva (Caldeira \& Perez, 2008), o teste de condutividade elétrica não foi adequado para avaliar a qualidade fisiológica durante o armazenamento.

Verificou-se diminuição dos valores de $\mathrm{CE}$ conforme se aumentou o volume de água. Para a maioria das combinações entre quantidade de sementes e volume de água, houve aumento na CE com o aumento do tempo de embebição, corroborando com os resultados de Torres et al. (1998), os quais também observaram aumento constante dos lixiviados conforme se aumentou o tempo de embebição das sementes.

Flávio \& Paula (2010), com sementes de Dictyoloma vandellianum, verificaram maiores valores de condutividade elétrica com o aumento no tempo de embebição, nos menores volumes de água e com menor número de sementes, diferindo deste trabalho em relação ao número de sementes, o qual 
Tabela 2. Valores de condutividade elétrica $\left(\mu \mathrm{S} . \mathrm{cm}^{-1} \cdot \mathrm{g}^{-1}\right)$ para três sublotes de sementes de B. virgilioides (1 - amarela; 2 - alaranjada; 3 - vermelha/preta), divididas em 25, 50 e 100 sementes, e acondicionadas em 25, 50 e 100 mL de água destilada por diferentes tempos de embebição

Table 2. Electrical conductivity values $\left(\mu \mathrm{S} . \mathrm{cm}^{-1} \cdot \mathrm{g}^{-1}\right)$ for three batches of seeds of B. virgilioides (1 - yellow, 2 - orage, 3 - red/black), divided into 25, 50 and 100 seeds and packed in 25, 50 and $100 \mathrm{~mL}$ of distilled water for different soaking times.

\begin{tabular}{|c|c|c|c|c|c|c|c|c|c|}
\hline \multirow[b]{2}{*}{$\begin{array}{l}\text { Tempo } \\
\text { (horas) }\end{array}$} & \multicolumn{3}{|c|}{25 sementes } & \multicolumn{3}{|c|}{50 sementes } & \multicolumn{3}{|c|}{100 sementes } \\
\hline & Amarela & Alaranjada & $\begin{array}{c}\text { Vermelha/ } \\
\text { Preta }\end{array}$ & Amarela & Alaranjada & $\begin{array}{c}\text { Vermelha/ } \\
\text { Preta } \\
\end{array}$ & Amarela & Alaranjada & $\begin{array}{c}\text { Vermelha/ } \\
\text { Preta } \\
\end{array}$ \\
\hline & \multicolumn{9}{|c|}{$25 \mathrm{~mL}$ de água } \\
\hline 12 & $32,5 \mathrm{cD}$ & $77,2 \mathrm{bE}$ & $275,8 \mathrm{aD}$ & $30,3 \mathrm{cD}$ & $66,9 \mathrm{bD}$ & $198,2 \mathrm{aD}$ & $26,5 \mathrm{cD}$ & $55,3 \mathrm{bD}$ & $210,9 \mathrm{aE}$ \\
\hline 24 & $36,6 \mathrm{cD}$ & $90,9 \mathrm{bD}$ & $394,9 \mathrm{aC}$ & $36,5 \mathrm{cC}$ & 88,1 bC & $293,3 \mathrm{aC}$ & $35,9 \mathrm{cC}$ & $77,3 \mathrm{bC}$ & $297,6 \mathrm{aD}$ \\
\hline 36 & $39,6 \mathrm{cCD}$ & 109,4 bCD & $454,6 \mathrm{aBC}$ & $36,8 \mathrm{cC}$ & $95,1 \mathrm{bBC}$ & $344,1 \mathrm{aBC}$ & $40,5 \mathrm{cC}$ & $85,9 \mathrm{bC}$ & $378,6 \mathrm{aC}$ \\
\hline 48 & $46,6 \mathrm{cBC}$ & $120,5 \mathrm{bBC}$ & $522,3 \mathrm{aAB}$ & $44,3 \mathrm{cB}$ & $106,5 \mathrm{bB}$ & $349,7 \mathrm{aB}$ & $46,6 \mathrm{cB}$ & $98,4 \mathrm{bB}$ & $450,7 \mathrm{aB}$ \\
\hline 60 & $51,4 \mathrm{cAB}$ & $134,6 \mathrm{bB}$ & 549,7 aA & $49,7 \mathrm{cAB}$ & $111,3 \mathrm{bAB}$ & $436,7 \mathrm{aA}$ & $50,7 \mathrm{cB}$ & $108,6 \mathrm{bAB}$ & $483,5 \mathrm{aAB}$ \\
\hline 72 & $56,3 \mathrm{cA}$ & $158,6 \mathrm{bA}$ & $607,6 \mathrm{aA}$ & $57,5 \mathrm{cA}$ & $127,7 \mathrm{bA}$ & $478,2 \mathrm{aA}$ & $60,2 \mathrm{cA}$ & $111,35 \mathrm{bA}$ & $526,5 \mathrm{aA}$ \\
\hline \multicolumn{10}{|c|}{$50 \mathrm{~mL}$ de água } \\
\hline 12 & $17,0 \mathrm{cD}$ & $35,3 \mathrm{bD}$ & $145,0 \mathrm{aD}$ & $12,2 \mathrm{cD}$ & $29,8 \mathrm{bD}$ & $124,1 \mathrm{aD}$ & $12,7 \mathrm{cF}$ & $35,0 \mathrm{bF}$ & $107,3 \mathrm{aF}$ \\
\hline 24 & $21,1 \mathrm{cC}$ & $50,1 \mathrm{bC}$ & $243,8 \mathrm{aC}$ & $16,1 \mathrm{cC}$ & $42,0 \mathrm{bC}$ & $192,0 \mathrm{aC}$ & $15,7 \mathrm{cE}$ & $47,2 \mathrm{bE}$ & $148,8 \mathrm{aE}$ \\
\hline 36 & $23,3 \mathrm{cC}$ & $52,0 \mathrm{bC}$ & $305,6 \mathrm{aB}$ & $17,3 \mathrm{cBC}$ & $45,4 \mathrm{bBC}$ & $230,2 \mathrm{aB}$ & $17,7 \mathrm{cD}$ & $55,6 \mathrm{bD}$ & $186,5 \mathrm{aD}$ \\
\hline 48 & $28,6 \mathrm{cB}$ & $59,5 \mathrm{bBC}$ & $322,7 \mathrm{aB}$ & $18,1 \mathrm{cBC}$ & $51,7 \mathrm{bAB}$ & $268,7 \mathrm{aAB}$ & $20,6 \mathrm{cC}$ & $65,8 \mathrm{bC}$ & $213,4 \mathrm{aC}$ \\
\hline 60 & $31,0 \mathrm{cAB}$ & $66,2 \mathrm{bAB}$ & $353,1 \mathrm{aAB}$ & $20,3 \mathrm{cAB}$ & $53,7 \mathrm{bAB}$ & $288,5 \mathrm{aA}$ & $23,1 \mathrm{cB}$ & $73,1 \mathrm{bB}$ & $229,3 \mathrm{aB}$ \\
\hline 72 & $36,5 \mathrm{cA}$ & $74,3 \mathrm{bA}$ & 387,4 aA & $21,8 \mathrm{cA}$ & $61,3 \mathrm{bA}$ & $315,0 \mathrm{aA}$ & $26,9 \mathrm{cA}$ & $81,7 \mathrm{bA}$ & $242,2 \mathrm{aA}$ \\
\hline \multicolumn{10}{|c|}{$100 \mathrm{~mL}$ de água } \\
\hline 12 & $11,6 \mathrm{cE}$ & $19,5 \mathrm{bE}$ & $55,1 \mathrm{aE}$ & $9,8 \mathrm{cD}$ & $17,9 \mathrm{bE}$ & $51,3 \mathrm{aD}$ & $5,9 \mathrm{cD}$ & $15,8 \mathrm{bD}$ & $50,9 \mathrm{aD}$ \\
\hline 24 & $14,2 \mathrm{cD}$ & $26,1 \mathrm{bD}$ & $81,0 \mathrm{aD}$ & $14,2 \mathrm{cC}$ & $26,5 \mathrm{bD}$ & $80,3 \mathrm{aC}$ & $7,3 \mathrm{cC}$ & $23,0 \mathrm{bC}$ & $78,1 \mathrm{aC}$ \\
\hline 36 & $14,6 \mathrm{cCD}$ & $28,6 \mathrm{bCD}$ & $93,8 \mathrm{aCD}$ & $15,7 \mathrm{cC}$ & $32,1 \mathrm{bC}$ & $96,8 \mathrm{aB}$ & $7,9 \mathrm{cC}$ & $26,8 \mathrm{bC}$ & $98,4 \mathrm{aC}$ \\
\hline 48 & $16,6 \mathrm{cBC}$ & $31,3 \mathrm{bBC}$ & $100,1 \mathrm{aBC}$ & $17,8 \mathrm{cB}$ & $37,7 \mathrm{bB}$ & $108,2 \mathrm{aAB}$ & $9,8 \mathrm{cB}$ & $31,4 \mathrm{bB}$ & $112,3 \mathrm{aB}$ \\
\hline 60 & $18,0 \mathrm{cB}$ & 34,9 bB & $108,7 \mathrm{aB}$ & $18,4 \mathrm{cAB}$ & $35,2 \mathrm{bBC}$ & $113,0 \mathrm{aA}$ & $10,9 \mathrm{cAB}$ & $34,8 \mathrm{bAB}$ & $124,7 \mathrm{aAB}$ \\
\hline 72 & $20,5 \mathrm{cA}$ & $38,7 \mathrm{bA}$ & $116,8 \mathrm{aA}$ & $21,1 \mathrm{cA}$ & $46,1 \mathrm{bA}$ & $123,8 \mathrm{aA}$ & $12,5 \mathrm{cA}$ & $36,6 \mathrm{bA}$ & 137,9 aA \\
\hline
\end{tabular}

Para cada combinação entre quantidade de sementes e volume de água, as médias seguidas pelas mesmas letras minúsculas na linha (sublotes) e maiúsculas na coluna (tempos de embebição) não diferem estatisticamente pelo teste de Tukey a 5\% de probabilidade.

foi verificado em alguns tratamentos, mas não foi padrão para todos.

As médias dos coeficientes de variação apresentadas na Tabela 3 denotam que quanto maior o volume de água e quanto menores forem as quantidades de sementes, maior será a variação nos valores de condutividade elétrica, ou seja, quanto mais diluída estiver a amostra, maior será o coeficiente de variação. Isto também foi observado por Santos \& Paula (2005) e Ataide et al. (2012), em que o coeficiente de variação foi menor nos tratamentos com maiores quantidades de sementes.

Como pode ser observado nas Figuras 1, 2 e 3, o coeficiente de determinação $\left(\mathrm{R}^{2}\right)$ variou de 0,9198 a 0,9994, indicando que menos de $9 \%$ da variabilidade do teste de condutividade elétrica não pode ser explicada pelas variáveis estudadas.
Para as sementes embebidas em $25 \mathrm{~mL}$ de água, em todas as combinações foi possível observar um aumento nos valores de CE conforme se aumentou o tempo de embebição, sendo possível diferenciar os três sublotes em níveis diferentes de vigor desde 12 horas de embebição.

Conforme se observa na Tabela 3 , no volume de $25 \mathrm{~mL}$, os valores foram significativos a $1 \%$ de probabilidade e obtiveram-se os valores mais baixos de coeficiente de variação. Neste volume de água a quantidade de lixiviados foi maior, possibilitando uma melhor diferenciação dos sublotes. Apesar disso, a pequena quantidade de água dificulta a leitura da solução de embebição, exigindo que o frasco seja inclinado para que o eletrodo fique totalmente imerso na solução. Note-se, também, que mesmo utilizando frascos com tampa, com o passar do tempo, pode haver evaporação da solução, 
Tabela 3. Valores dos quadrados médios (QM) e coeficiente de variação (CV\%) da condutividade elétrica em sementes de $B$. virgilioides para cada combinação entre quantidades de sementes e volumes de água.

Table 3. Presents the Mean square (QM) and coefficient of variation (CV\%) of electrical conductivity in seeds of $B$. virgilioides, for each combination of quantities of seeds and water volumes.

\begin{tabular}{|c|c|c|c|c|c|c|c|}
\hline & \multicolumn{2}{|c|}{25 sementes } & \multirow{2}{*}{\multicolumn{2}{|c|}{$\begin{array}{c}50 \text { sementes } \\
25 \mathrm{~mL}\end{array}$}} & \multicolumn{2}{|c|}{100 sementes } & \multirow{3}{*}{$\begin{array}{l}\text { Média } \\
\text { CV\% }\end{array}$} \\
\hline & \multirow[b]{2}{*}{$\mathbf{Q M}$} & \multirow[b]{2}{*}{ CV\% } & & & \multirow[b]{2}{*}{ QM } & & \\
\hline & & & $\mathbf{Q M}$ & CV\% & & CV\% & \\
\hline Sublotes & $10,79^{* *}$ & 25,72 & $7,63^{* *}$ & 12,79 & $8,52^{\star \star}$ & 11,13 & 16,55 \\
\hline Tempos & $0,19^{* *}$ & 2,06 & $0,22^{\star *}$ & 2,03 & $0,29^{* *}$ & 1,62 & 1,90 \\
\hline Sublotes $\times$ Tempos & $0,006^{* *}$ & & \multicolumn{2}{|l|}{$0,005^{\star *}$} & \multicolumn{2}{|l|}{$0,004^{\star \star}$} & \\
\hline \multicolumn{8}{|c|}{$50 \mathrm{~mL}$} \\
\hline Sublotes & $10,83^{* *}$ & 22,42 & $11,54^{\star \star}$ & 16,55 & $8,67^{* *}$ & 18,33 & 19,1 \\
\hline Tempos & $0,28^{\star *}$ & 2,50 & $0,22^{\star *}$ & 2,56 & $0,28^{* *}$ & 2,17 & 2,41 \\
\hline Sublotes $\times$ Tempos & $0,009^{* *}$ & & $0,008^{\star \star}$ & & $0,002^{\text {ns }}$ & & \\
\hline \multicolumn{8}{|c|}{$100 \mathrm{~mL}$} \\
\hline Sublotes & $5,24^{\star \star}$ & 22,04 & $5,77^{* *}$ & 28,49 & $9,79^{* *}$ & 15,67 & 22,07 \\
\hline Tempos & $0,18^{* *}$ & 3,02 & $0,30^{* *}$ & 2,27 & $0,34^{* \star}$ & 4,90 & 3,40 \\
\hline Sublotes $\times$ Tempos & $0,003^{\text {ns }}$ & & $0,003^{*}$ & & $0,005^{\text {ns }}$ & & \\
\hline \multirow{2}{*}{ Média CV\% } & & 23,40 & & 19,28 & & 15,04 & \\
\hline & & 2,53 & & 2,29 & & 2,90 & \\
\hline
\end{tabular}

${ }^{* *}$ Significativo a $1 \% .{ }^{*}$ Significativo a $5 \%$. ${ }^{\text {ns }}$ Não significativo.

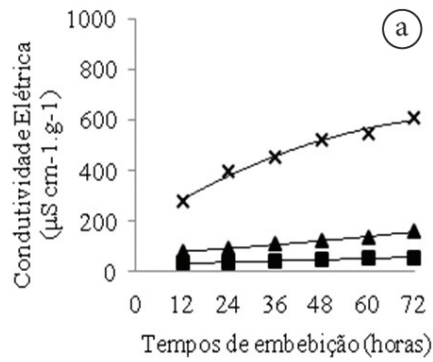

CE (Lote 1) $=0,0014 x^{2}+0,29 x+28,64 R^{2}=0,9933$ CE (Lote 1) $00,0014 \mathrm{x}^{2}+0,29 \mathrm{X}+28,64 \quad \mathrm{R}^{2}=0,9933$ CE (Lote 2) $=0,0043 \mathrm{x}^{2}+0,9491 \mathrm{x}+66,029 \quad \mathrm{R}^{2}=0,9922$

- Lote 1

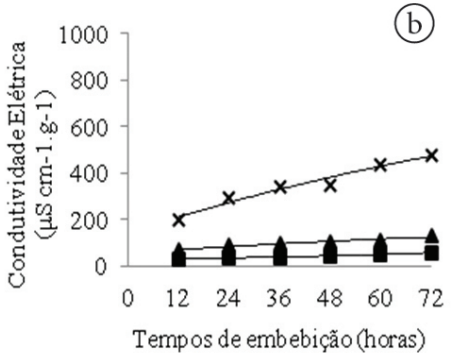

CE (Tote 1) $=0,0035 x^{2}+0.1428 x+28,893 R^{2}=0,9822$ CE (Lote 1) $=0,0035 x^{2}+0,1428 x+28,893 \quad R^{2}=0,9822$ CE (Lete 2) $=0,004 x^{2}+1,256 x+55,334 \quad R^{2}=0,9703$

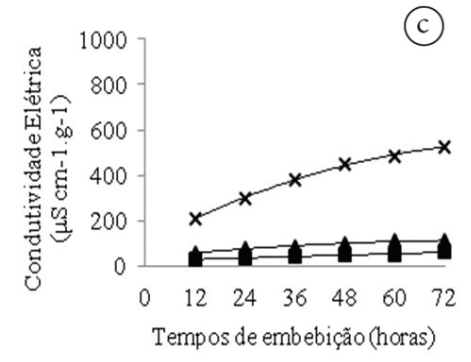

CE (Lote 1$)=-0,0001 \mathrm{x}^{2}+0,5321 \mathrm{x}+21,328 \quad \mathrm{R}^{2}=0,9845$ CE (Lote 2$)=-0,0112 \mathrm{x}^{2}+1,8581 \mathrm{x}+35,808 \quad \mathrm{R}^{2}=0,9907$ $C E($ Lote 3$)=-0,051 \mathrm{x}^{2}+9,5415 \mathrm{x}+101,98 \mathrm{R}^{2}=0,998$

Figura 1. Valores de condutividade elétrica $\left(\mu \mathrm{S} \mathrm{cm}^{-1} \cdot \mathrm{g}^{-1}\right)$ para três sublotes de sementes de B. virgilioides. Amostras com 25 (A), 50 (B) e 100 sementes (C) imersas em $25 \mathrm{~mL}$ de água destilada por diferentes tempos de embebição. Figure 1. Values of electrical conductivity $\left(\mathrm{mS} \mathrm{cm}^{-1} \cdot \mathrm{g}^{-1}\right)$ for three batches of seeds of $B$. virgilioides. Samples with 25 (A), 50 (B) and 100 seeds (C) immersed in $25 \mathrm{~mL}$ of distilled water for different immersion times.

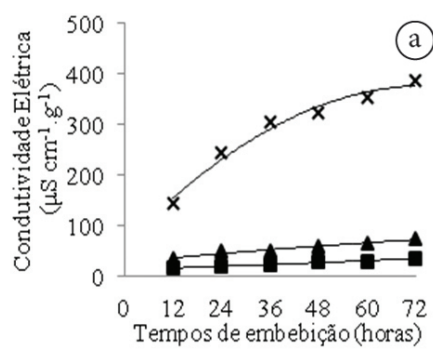

CE (Lote 1) $=0,0009 \mathrm{x}^{2}+0,2383 \mathrm{x}+14,244 \quad \mathrm{R}^{2}=0,99$ $\begin{array}{ll}\mathrm{CE}\left(\text { Lote 2) }=-0,0018 \mathrm{x}^{2}+0,7506 \mathrm{x}+28,694\right. & \mathrm{R}^{2}=0,9684 \\ \mathrm{CE} \text { (Lote 3) }=-0,0556 \mathrm{x}^{2}+8,3747 \mathrm{x}+62,541 \quad \mathrm{R}^{2}=0,9771\end{array}$

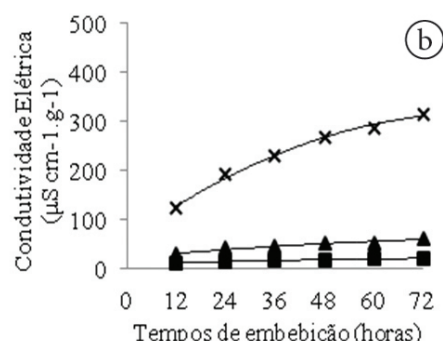

Tempos de emb ebição (horas)

$\mathrm{CE}$ (Lote 1) $=-0,1384 \mathrm{x}^{2}+2,717 \mathrm{x}+10,231 \quad \mathrm{R}^{2}=0,9638$ CE (Lote 2) $=-0,51 \mathrm{x}^{2}+92651 \mathrm{x}+22,632 \mathrm{R}^{2}=0,9672$ $\mathrm{CE}($ Lote 3$)=-5,0104 \mathrm{x}^{2}+71,711 \mathrm{x}+61,426 \quad \mathrm{R}^{2}=0,9951$

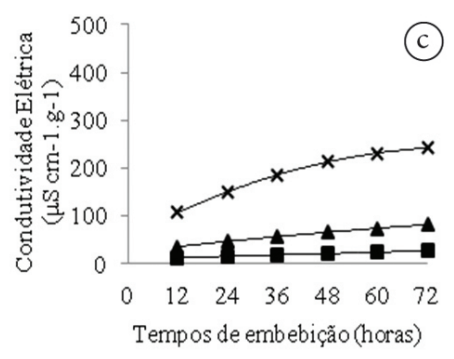

CE (Lote 1) $=0,1146 \mathrm{x}^{2}+1,9498 \mathrm{x}+10,882 \quad \mathrm{R}^{2}=0,9963$ $\begin{array}{ll}\text { CE }(\text { Lote 2) })=-0,4052 \mathrm{x}^{2}+12,024 \mathrm{x}+23,823 & \mathrm{R}^{2}=0,998 \\ \text { CE }(\text { Lote } 3)=-4,1048 \mathrm{x}^{2}+55,672 \mathrm{x}+55,315 & \mathrm{R}^{2}=0,999\end{array}$

- Lote 1

$\Delta$ Lote $2 \times$ Lote 3

Linha de Tendência

Figura 2. Valores de condutividade elétrica $\left(\mu \mathrm{S} \mathrm{cm}^{-1} \cdot \mathrm{g}^{-1}\right)$ para três sublotes de sementes de B. virgilioides. Amostras com 25 (A), 50 (B) e 100 sementes (C) imersas em $50 \mathrm{~mL}$ de água destilada por diferentes tempos de embebição. Figure 2. Values of electrical conductivity $\left(\mathrm{mS} \mathrm{cm}^{-1} \cdot \mathrm{g}^{-1}\right)$ for three batches of seeds of B. virgilioides. Samples with 25 (A), 50 (B) and 100 seeds (C) immersed in $50 \mathrm{~mL}$ of distilled water for different immersion times. 


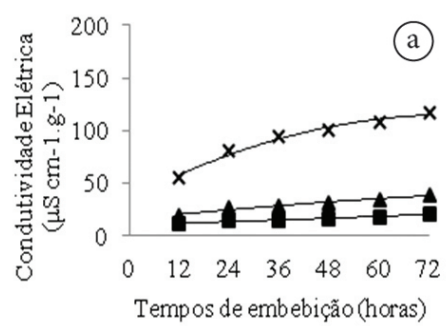

CE (Lote 1) $=0,0605 \mathrm{x}^{2}+1,24 \mathrm{x}+10,677 \mathrm{R}^{2}=0.9793$ $\mathrm{CE}$ (Lote 2) $=-0,2011 \mathrm{x}^{2}+4,9435 \mathrm{x}+15,554 \quad \mathrm{R}^{2}=0,9835$

Lote 1

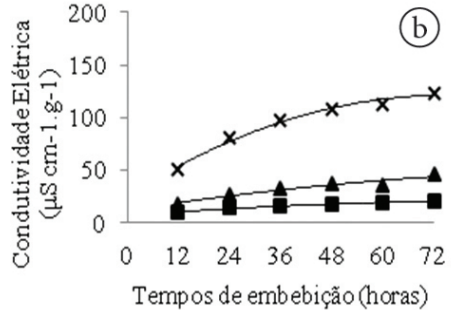

CE (Lote 1$)=-0,2159 \mathrm{x}^{2}+3,5492 \mathrm{x}+7,007 \quad \mathrm{R}^{2}=0,968$ CE (Lote 2) $=-0,3816 \mathrm{x}^{2}+7,6033 \mathrm{x}+11,753 \quad \mathrm{R}^{2}=0,919$ CE(Lote 2$)=-0,3816 \mathrm{x}^{2}+7,6033 \mathrm{x}+11,7,33 \mathrm{R}^{2}=0,9898$

Lote $2 \times$ Lote 3

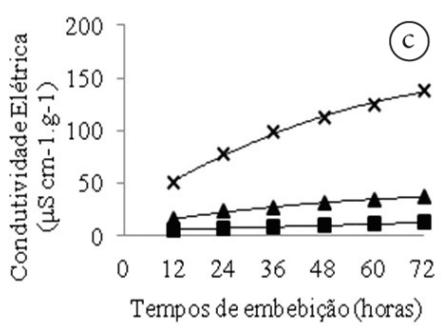

CE $($ Lote 1$)=0,0473 \mathrm{x}^{2}+0,9696 \mathrm{x}+4,947 \quad \mathrm{R}^{2}=0,9913$ $\mathrm{CE}($ Lote 2$)=-0,5179 \mathrm{x}^{2}+7,7504 \mathrm{x}+8,806 \quad \mathrm{R}^{2}=0,9968$ CE (Lote 3) $=-1,8225 \mathrm{x}^{2}+29,582 \mathrm{x}+24,484 \mathrm{R}^{2}=0,9969$

Figura 3. Valores de condutividade elétrica $\left(\mu \mathrm{S} \mathrm{cm}^{-1} \cdot \mathrm{g}^{-1}\right)$ para três sublotes de sementes de B. virgilioides. Amostras com 25 (A), 50 (B) e 100 sementes (C) imersas em $100 \mathrm{~mL}$ de água destilada por diferentes tempos de embebição. Figure 3. Values of electrical conductivity $\left(\mathrm{mS} \mathrm{cm}^{-1} \cdot \mathrm{g}^{-1}\right)$ for three batches of seeds of B. virgilioides. Samples with 25 (A), 50 (B) and 100 seeds (C) immersed in $100 \mathrm{~mL}$ of distilled water for different immersion times.

diminuindo a coluna d'água, conforme foi verificado por Santos \& Paula (2005)

Diante destas condições, não se recomenda o uso de $25 \mathrm{~mL}$ para o teste de condutividade elétrica em sementes de B. virgilioides, em frascos com $6 \mathrm{~cm}$ de diâmetro de base. Sugere-se estudar o uso de recipientes com diâmetro de base menor do que os utilizados neste estudo, mesmo que este valor esteja dentro do recomendado por Vieira \& Krzyzanowski (1999), que é de 6 a $7 \mathrm{~cm}$.

$\mathrm{O}$ volume de $50 \mathrm{~mL}$ de água foi eficiente na diferenciação dos sublotes para todas as quantidades de sementes analisadas, sendo possível observar diferenças de vigor desde 12 horas de embebição. Os valores de coeficiente de variação foram intermediários e os valores $\mathrm{R}^{2}$ foram todos acima de 0,96 , sendo que, na quantidade de 100 sementes, encontrou-se o maior valor do trabalho: 0,999. Esta quantidade de sementes também aumenta a concentração de eletrólitos na solução de embebição, sendo recomendada para estudos de condutividade elétrica em sementes de B. virgilioides. Porém, em estudos posteriores, se a amostra para análise das sementes for pequena, acredita-se que a diminuição na quantidade de sementes para 25 ou 50 não acarretará diferenças significativas no valor final da condutividade elétrica.

No volume de $100 \mathrm{~mL}$, verificaram-se os menores valores de $\mathrm{CE}$, devido à maior diluição dos íons na solução de embebição. Esta diluição pode ter causado aumento no coeficiente de variação destes tratamentos, como também diminuição do $\mathrm{R}^{2}$, que chegou a 0,92 em um dos tratamentos. Desta forma, mesmo sendo possível a diferenciação dos sublotes neste volume de água, não se recomenda a sua utilização em testes posteriores. Esta diminuição nos valores de CE com o aumento da quantidade de água também foi observada por Ataide et al. (2012) e Pereira \& Martins Filho (2012).

Para Flávio \& Paula (2010), no volume de $100 \mathrm{~mL}$, houve pouca diferença nos padrões de lixiviação no período inicial de embebição em sementes de Dictyoloma vandellianum, independentemente do número utilizado, indicando que se devem utilizar menores quantidades de sementes para obter resultados mais rápidos. Isso diverge do que foi observado neste estudo, pois mesmo no volume de $100 \mathrm{~mL}$, que apresentou diluição, foi possível diferenciar os sublotes de sementes segundo níveis de qualidade.

Em relação ao tempo de embebição, os valores de condutividade elétrica não se estabilizaram ao longo das medições, uma vez que, na maioria dos tratamentos, observou-se aumento significativo do valor de CE até as 72 horas. De acordo com Rosa et al. (2000), a liberação inicial de eletrólitos é intensa, tanto pelas sementes vigorosas quanto pelas deterioradas, sendo difícil diferenciar lotes no começo da embebição. Na medida em que as sementes vigorosas reorganizam mais rapidamente suas membranas, a sua liberação de íons se estabiliza, sendo possível a diferenciação dos lotes em níveis de qualidade. Contudo, neste estudo, a diferenciação dos sublotes já foi observada desde a primeira 
medição, com 12 horas, sendo desnecessário esperar a estabilização dos valores de CE.

Observou-se aumento crescente nos valores de condutividade elétrica, conforme se aumentou o tempo de embebição das sementes. Isto não foi verificado nos trabalhos de Ataide et al. (2012) e Pereira \& Martins Filho (2012), nos quais se observou variação irregular entre os períodos de avaliação. De acordo com Flávio \& Paula (2010), há a suspeita de reabsorção de eletrólitos pelas sementes, de forma a se atingir um equilíbrio entre a semente e a solução circundante, o que constitui um objeto de estudos futuros.

A diferenciação dos sublotes desde as 12 horas de embebição na maioria dos tratamentos pode ser decorrente do tamanho das sementes. B. virgilioides apresentou peso de mil sementes igual a 25,46 g, valor semelhante ao encontrado por Dutra et al. (2007) em sementes de Senna siamea (25,4 g), em que, com 6 horas, foi possível diferenciar os lotes em níveis diferentes de qualidade fisiológica. Ambas as espécies pertencem à família Fabaceae, logo podem apresentar comportamentos semelhantes de embebição, sugerindo que o teste de CE em sementes de B. virgilioides pode ser realizado em menor tempo.

De acordo com a recomendação feita por Vieira \& Krzyzanowski (1999), 24 horas é o período ideal de embebição, pois possibilita uma separação mais eficiente de lotes com diferença estreita de vigor; este período é melhor, também, porque facilita a organização das atividades nos laboratórios de análises. Desta forma, recomenda-se a utilização de 24 horas de embebição para o teste de CE em sementes de $B$. virgilioides.

A partir dos resultados obtidos, foi possível diferenciar os sublotes de B. virgilioides em três diferentes graus de qualidade fisiológica. As sementes de coloração amarela possuem maior vigor, seguidas pelas sementes de coloração alaranjada (vigor intermediário) e pelas sementes vermelhas ou pretas, com menor vigor. Desta forma, sugere-se que, em estudos futuros, envolvendo as sementes de $B$. virgilioides, seja preferencial o uso de sementes com coloração amarela do tegumento, como também se sugere a eliminação das sementes vermelhas ou pretas dos lotes de sementes.

\section{CONCLUSÕES}

O teste de condutividade elétrica é eficiente na determinação da qualidade fisiológica de sementes de $B$. virgilioides. Recomenda-se a utilização de 25, 50 ou 100 sementes embebidas em $50 \mathrm{~mL}$ de água por 24 horas, na temperatura constante de $25^{\circ} \mathrm{C}$.

Confirmou-se a hipótese de que a coloração do tegumento interfere na qualidade fisiológica das sementes. Foi possível diferenciar os sublotes em três graus de qualidade fisiológica, sendo as sementes de coloração amarela com maior vigor.

\section{STATUS DA SUBMISSÃO}

Recebido: 16/08/2013

Aceito: $12 / 02 / 2014$

Publicado: 31/02/2014

\section{AUTOR(ES) PARA CORRESPONDÊNCIA}

\section{Samanta Jaqueline Dalanhol}

Departamento de Botânica, Universidade Estadual Paulista - UNESP, CEP 18618-970, Botucatu, SP, Brasil e-mail: dalanholsj@gmail.com

\section{REFERÊNCIAS}

Araujo RF, Zonta JB, Araújo EF, Donzeles SML, Costa GM. Teste de condutividade elétrica para sementes de pinhão-manso (Jatropha curcas L.). Idesia 2011; 29: 79-86. http://dx.doi.org/10.4067/S071834292011000200010

Ataide GM, Flôres AV, Borges EEL, Resende RT. Adequação da metodologia do teste de condutividade elétrica para sementes de Pterogyne nitens Tull. Revista Brasileira de Ciências Agrárias 2012; 7: 635-640. http:// dx.doi.org/10.5039/agraria.v7i4a1688

Borghetti F, Ferreira AG. Interpretação de resultados de germinação. In: Ferreira AG, Borghetti F. Germinação: do básico ao aplicado. Porto Alegre: Artmed; 2004. p. 209-222.

Brasil. Ministério da Agricultura, Pecuária e Abastecimento. Regras para análise de sementes. Brasília: Ministério da Agricultura; 2009. 395 p.

Caldeira SF, Perez SCJGA. Qualidade de diásporos de Myracrodruon urundeuva Fr. All. armazenados sob diferentes condições. Revista Brasileira de Sementes 
2008; 30: 185-194. http://dx.doi.org/10.1590/S010131222008000300025

Carvalho PER. Espécies arbóreas brasileiras. Brasília: Embrapa Informação Tecnológica; 2006.2 v.627@@p.

Dutra AS, Medeiros Filho S, Diniz FO. Teste de condutividade elétrica em sementes de Senna siamea (Lam.) H.S. Irwin \& Barneby. Revista Ciência Agronômica 2007; 38: 280-285.

Flávio JJP, Paula RC. Testes de envelhecimento acelerado e de condutividade elétrica em sementes de Dictyoloma vandellianum A. Juss. Scientia Forestalis 2010; 38: 391399.

Fowler JAP, Martins EG. Manejo de sementes de espécies florestais. Colombo: Embrapa Florestas; 2001. 71 p. Documentos, n. 58.

Gonçalves EP, Paula RC, Desmatlê MESP. Testes de vigor em sementes de Guazuma ulmifolia Lam. Semina: Ciências Agrárias 2008; 29: 265-276.

Gonzales JLS, Paula RC, Valeri SV. Teste de condutividade elétrica em sementes de Albizia hassleri (Chodat) Burkart. Fabaceae - Mimosoideae. Revista Árvore 2009; 33: 625-634. http://dx.doi.org/10.1590/ S0100-67622009000400005

Hadas A. Water uptake and germination of leguminous seeds under changing external water potential in osmotic solution. Journal of Experimental Botany 1976; 27: 480-489. http://dx.doi.org/10.1093/jxb/27.3.480

Labouriau LG. A germinação das sementes. Washington: Organização dos Estados Americanos; 1983. 174 p.

Lorenzi H. Árvores brasileiras: manual de identificação e cultivo de plantas arbóreas nativas do Brasil. 5. ed. Nova Odessa: Instituto Plantarum; 2008. 384 p.

Maguire JD. Speed of germination aid in selection and evaluation for seedling emergence and vigor. Crop Science 1962; 2: 176-177. http://dx.doi.org/10.2135/cro psci1962.0011183X000200020033x

Marcos Filho J. Fisiologia de sementes de plantas cultivadas. Piracicaba: FEALQ; 2005. 495 p.
Marques MA, Paula RC, Rodrigues JD. Adequação do teste de condutividade elétrica para determinar a qualidade fisiológica de sementes de jacarandá-dabahia (Dalbergia nigra (Vell.) Fr.All. ex Benth). Revista Brasileira de Sementes 2002; 24: 271-278.

Matos E, Queiroz LP. Árvores para Cidades. Salvador: Ministério Público do Estado da Bahia: Solisluna; 2009. $340 \mathrm{p}$.

Pereira MD, Martins Filho, S. Adequação da metodologia do teste de condutividade elétrica para sementes de cubiu (Solanum sessiliflorum DUNAL). Revista Agrarian 2012; 5: 93-98.

Rosa SDVF, Pinho EVRV, Vieira MGGC, Veiga RD. Eficácia do teste de condutividade elétrica para uso em estudos de danos de secagem em sementes de milho. Revista Brasileira de Sementes 2000; 22: 54-63.

Santos SRG, Paula RC. Teste de condutividade elétrica para avaliação da qualidade fisiológica de sementes de Sebastiania commersoniana (Bail) Smith \& Downs - Euphorbiaceae. Revista Brasileira de Sementes 2005; 27: 136-145. http://dx.doi.org/10.1590/S010131222005000200020

Silva A, Perez SCJGA, Paula RC. Qualidade fisiológica de sementes de Psidium cattleianum Sabine acondicionadas e armazenadas em diferentes condições. Revista Brasileira de Sementes 2011; 33: 197-206. http:// dx.doi.org/10.1590/S0101-31222011000200001

Tillmann MAA, Miranda DM. Módulo 4: Análise de sementes. In: Curso de Ciência e Tecnologia de Sementes. Brasília: ABEAS; Pelotas: Universidade Federal de Pelotas; 2007. $102 \mathrm{p}$

Torres SB, Caseiro RF, Rodo AB, Marcos Filho J. Testes de vigor em sementes de maxixe (Cucumis anguria $\mathrm{L}$.) com ênfase ao teste de condutividade elétrica. Revista Brasileira de Sementes 1998; 20: 241-244.

Vieira RD, Krzyzanowski FC. Teste de Condutividade Elétrica. In: Krzyzanowski FC, Vieira RD, França JB No. Vigor de Sementes: Conceitos e Testes. Londrina: ABRATES; 1999. p. 4-26. 